\title{
¿s Research Square \\ Detection of Subclinical Keratoconus Using a Novel Combined Tomographic and Biomechanical Model Based on an Automated Decision Tree
}

\author{
Peng Song \\ People's Hospital of Zhengzhou University \\ Shengwei Ren \\ People's Hospital of Zhengzhou University \\ Yu Liu \\ Central South University \\ Pei Li \\ Central South University \\ Qingyan Zeng ( $\sim$ zengqingyan1972@163.com) \\ Central South University
}

\section{Research Article}

Keywords: Detection of Subclinical Keratoconus, Tomographic, Biomechanical Model, Automated Decision Tree, eyes, algorithms, biomechanical imaging

Posted Date: November 19th, 2021

DOI: https://doi.org/10.21203/rs.3.rs-1056294/v1

License: (1) This work is licensed under a Creative Commons Attribution 4.0 International License. Read Full License

Version of Record: A version of this preprint was published at Scientific Reports on March 29th, 2022. See the published version at https://doi.org/10.1038/s41598-022-09160-6. 


\section{Abstract}

The aim of this study was to develop a predictive model for subclinical keratoconus (SKC) based on decision tree (DT) algorithms. A total of 194 eyes (including 105 normal eyes and 89 SKC) were included in the double-center retrospective study. Data were separately used for training and validation databases. The baseline variables were derived from tomography and biomechanical imaging. DT models were generated in the training database using Chi-square automatic interaction detection (CHAID) and classification and regression tree (CART) algorithms. The discriminating rules of the CART model selected variables of the Belin/Ambrósio deviation (BAD-D), stiffness parameter at first applanation (SPA1), back eccentricity (Becc), and maximum pachymetric progression index in order, while the CHAID model selected BAD-D, deformation amplitude ratio, SPA1, and Becc. The CART model allowed discrimination between normal and SKC eyes with 92.2\% accuracy, which was higher than that of the CHAID model (88.3\%), BAD-D (82.0\%), Corvis biomechanical index (CBI, 77.3\%), and tomographic and biomechanical index (TBI, 78.1\%). The discriminating performance of the CART model was validated with $92.4 \%$ accuracy, while the CHAID model was validated with $86.4 \%$ accuracy in the validation database. Thus, the CART model using tomography and biomechanical imaging was an excellent model for SKC screening and provided easy-to-understand discriminating rules.

\section{Introduction}

Keratoconus $(\mathrm{KC})$ is a progressive sight-threatening ectatic corneal disease that is one of the most common causes of corneal blindness in adolescents. ${ }^{1}$ Due to the overlap in most parameters derived from topography and tomography imaging between subclinical keratoconus (SKC) and normal eyes (NE), identifying SKC remains one of the most challenging situations before making the decision to perform refractive surgery. ${ }^{2,3}$ In the field of SKC screening, establishing combinations or models based on different algorithms has attracted increasing attention.

Currently, the strict criteria that define SKC are not well established. The inclusion criteria of subjects influence the outcomes of predictors' discriminatory power in diagnosis studies. Previously, most studies only adopted topographic criteria to define the SKC, which may result in overestimation of the model's performance. ${ }^{4,5} \mathrm{Hwang}$ reported that a logistic regression model achieved amazing high accuracy $(A U C=1.0)$ in detecting the SKC with a normal topographic aspect. ${ }^{4}$ Ambrósio et al. introduced a new combined index, the tomographic and biomechanical index $(\mathrm{TBI})^{6}$, which was shown to exhibit a high AUC value of 0.985 in detecting SKC with a topography-based KISA\% index ${ }^{7}$ of less than $60 \%$. However, Steinberg et al. ${ }^{8}$ demonstrated that the discriminatory power of TBI decreased with an AUC of 0.825 in a validation research using the same inclusion and exclusion criteria as Ambrósio's study ${ }^{6}$. The results of the aforementioned studies appear to be encouraging; however, when evaluating the performance of a model, the distribution of variables within the studied population should be considered seriously. Although the first detectable sign of $\mathrm{KC}$ has not been defined definitively, previous studies highlight the importance of posterior surface abnormalities in SKC diagnosis. ${ }^{9,10}$ Bae suggested that the back elevation difference (BED) should be included in the criteria of SKC for the future. ${ }^{3}$ Therefore, further study should be performed to detect SKC defined by stricter criteria considering topography and posterior surface elevation.

Clinically, another troublesome situation in SKC diagnosis is choosing the most useful variables from vast amounts of data and interpreting the complex variables. In practice, the final decision before refractive surgery is often based on personal experience or proposed cutoffs of summary metrics in previous studies. Generally, an ideal model is easily interpretable, clinically credible, and statistically valid. A decision tree (DT) model represents a symbolic classification with "if-then rules", which could provide easier interpretation than that of purely quantitative models, such as neural networks and logistic regression. Based on the DT algorithm, Smadja analyzed the metrics only from the GALILEI Dual-Scheimpflug Analyzer and reported an easy-to-understand discriminating rule and its desired accuracy for SKC screening. ${ }^{5}$

The purpose of the present study was to develop easy-to-understand discriminating rules based on DT algorithms by overall analysis of the metrics from tomography and biomechanical imaging and evaluate the performance of DT models in detecting SKC defined with normal topography and BED.

\section{Materials And Methods}


This retrospective study was performed through a cooperative effort between the Wuhan Aier Hankou Eye Hospital (Wuhan, China) and Henan Eye Hospital (Zhengzhou, China). Our study adhered to the tenets of the Declaration of Helsinki and was separately approved by Institutional Review Boards from the Wuhan Aier Hankou Eye Hospital and the Henan Eye Hospital. Written informed consent was obtained from each participant.

One hundred ninety-four participants were included in this double-center retrospective study. A total of 128 participants (70 healthy and 58 with very asymmetric ectasia) were enrolled from the Hankou Aier Eye Hospital (training database), and 66 participants (35 healthy and 31 with very asymmetric ectasia) were enrolled from the Henan Eye Hospital (validation database). All patients underwent comprehensive ophthalmic examinations, including a subjective analysis with slit-lamp biomicroscopy by an experienced anterior segment expert and objective examinations (i.e., Corvis ST and Pentacam HR examinations).

The objective definitions for normal and KC were based on the well-established topographical keratoconus classification (TKC) index from topometric display and tomography imaging in the Pentacam system. ${ }^{3}$ An eye was diagnosed as having $\mathrm{KC}$ if it met with (1) a TKC index of greater than 0 and (2) at least one of the following signs: Vogt striae, Fleischer ring, Munson's sign, and focal thinning. The diagnosis of KC was only used to aid the definition of SKC and was not used for further analysis. The less affected fellow eye of a very asymmetric ectasia individual was diagnosed as having SKC if it met with (1) normal slit-lamp, (2) CDVA of 20/20 or better, (3) normal topographic aspect, a TKC index of $0,{ }^{11}$ the central mean keratometry value $<47.2 \mathrm{D}$ and I-S value $<1.40 \mathrm{D},{ }^{12}$ (4) BED $<12 \mu \mathrm{m}^{13}$. The NE definition was met with the same criteria as in SKC (both eyes of normal individuals met the criteria). All normal control patients underwent refractive surgery and had a 2-year follow-up without any evidence of ectatic corneal changes. Only one eye was randomly selected for further analysis.

For all eyes included in the study, no contact lenses had been worn for at least 4 (rigid contact lenses) or 2 (soft contact lenses) weeks prior to examination; no history of other eye diseases, no previous ocular surgery, and no use of topical medications other than artificial tears.

The techniques for Pentacam and Corvis ST analyses have been described previously. ${ }^{14,15}$ Not only the "QS" buttons from the Corvis ST and Pentacam HR read OK but also the measured area of Belin/Ambrósio Enhanced Ectasia was no smaller than $8 \mathrm{~mm}$, and the measurement was included in the study. All cases from each hospital had tomographic data masked for reevaluation by an anterior segment expert from the other center (SW and QY) to confirm recruitment.

Objectively, the variables analyzed included keratometry values, eccentricity, and astigmatism of the anterior and posterior surfaces; Kmax; the distance between any two points of the corneal apex (Apex), corneal thinnest corneal point (TCP), and pupil center point

(PCP) [including the distances (D) from the TCP to the Apex, $\mathrm{D}_{\text {TCP-Apex }}$ TCP to PCP, $\mathrm{D}_{\text {TCP-PCP; }}$ and PCP to Apex, $\mathrm{D}_{\mathrm{PCP}-\mathrm{Apex}}$; PPImax (maximum of pachymetric progression index); Belin/Ambrósio deviation index (BAD-D); BED; back eccentricity (Becc); index of surface variance (ISV); index of vertical asymmetry (IVA); index of height asymmetry (IHA); index of height decentration (IHD); keratoconus index (KI); central keratoconus index (CKI); deformation amplitude ratio (DA-ratio); integrated radius (Integ-R); stiffness parameter at first applanation (SPA1); Corvis biomechanical index (CBI); and TBI.

\section{Description and tree-growing criteria of both the CHAID and CART decision trees}

We used SPSS 22.0 software to generate DTs based on chi-square automatic interaction detection (CHAID) and classification and regression tree (CART) algorithms in the training database (schematic theory of the discriminating rule based on a DT is presented in Supplementary Figure 1). The tree-growing method of CHAID chooses the independent variable that has the strongest interaction with the dependent variable. Chi-square tests were used to determine node splitting and category merging. In the CHAID method, the most significant discriminant variable divides the entire sample into two or more subgroups, which are subsequently divided by the next selected variable. The significance level for node splitting in the CHAID model was a $P$-value less than 0.05 . The CART method splits the data into segments that are as homogeneous as possible with respect to the dependent variable in the tree growing procedure. A DT generated using CART is referred to as a binary tree. The CART-growing method attempts to maximize within-node homogeneity, and the Gini was selected to measure impurity at the split. The minimum change in improvement was set as 0.0001 . Furthermore, the pruning procedure was performed in the CART procedure to reduce overfitting. 


\section{Growth limits}

The maximum level of tree depth was set to 3 for CHAID and 5 for CART. The minimum number of cases was set to 10 for parent nodes and 5 for child nodes.

\section{Internal- and external-validation}

A 10 -fold cross-validation method was used for internal validation. The training database was split into 10 approximately equalsized folds, and trees were then produced 10 times. The final tree was selected to be the one with the smallest cross-validation estimate of error. The external validation procedure was performed based on the validation database from Henan Eye Hospital.

Statistical analyses were performed using SPSS 22.0 software and MedCalc software. The mean \pm standard deviation (SD) and range were used to describe the data. All data were analyzed using the Kolmogorov-Smirnov normality test and Levene's test for equal variances to choose the appropriate method. The differences between two groups were compared using the independent $t-$ test or Mann-Whitney U test. The DTs were generated based on the CHAID and CART methods. The receiver operating characteristic (ROC) curve was used to test the performance of the studied metrics and DT models in differentiating populations. DeLong's test was used for the pairwise comparisons of AUCs. $P<0.05$ (two-tailed) was considered statistically significant.

\section{Results}

The predictive accuracies of the CHAID model in the training database (CHAID-T) and CART model in the training database (CART-T) were $88.3 \%$ and $92.2 \%$, respectively (Table 1 ). The CART-T model had a higher sensitivity of $89.7 \%$ than the CHAID-T model ( $82.8 \%$ sensitivity) (Table 1). The most important predictors in the CHAID-T to discriminate NE and SKC included the BAD-D, DA-ratio, SPA1, and Becc (Figure 1 and Table 2), whereas that in CART-T included the BAD-D, SPA1, Becc, and PPImax (Figure 2 and Table 2). For both trees, the BAD-D was selected as the most discriminant variable to differentiate SKC and NE at the first split (with similar cutoff values of BAD-D: 1.53 in the CHAID-T and 1.635 in the CART-T). SPA1 was selected as the second most discriminant variable for differentiating SKC from NE (cutoff values: 92.2 in the CHAID-T and 89.25 in the CART-T). The external validation procedure revealed that the CHAID tree in the validation database (CHAID-V) had $86.4 \%$ accuracy and that the CART tree in the validation database (CART-V) had $92.4 \%$ accuracy (Table 1 ). The interpretation details of the discriminating rules of the CHAID-T and CART-T are summarized in Table 2, Figure 1, and Figure 2.

Table 1

The performance of CHAID and CART decision tree to discriminate SKC from NE

\begin{tabular}{|llllll|}
\hline Model & Eye number (NE vs SKC) & Accuracy (\%) & Youden & Sensitivity (\%) & Specificity (\%) \\
\hline CHAID-T & 70 vs 58 & 88.3 & 0.76 & 82.8 & 92.9 \\
\hline CART-T & 70 vs 58 & 92.2 & 0.84 & 89.7 & 94.3 \\
\hline CHAID-V & 35 vs 31 & 86.4 & 0.73 & 90.3 & 82.9 \\
\hline CART-V & 35 vs 31 & 92.4 & 0.85 & 90.3 & 94.3 \\
\hline $\begin{array}{l}\text { CHAID-T, CHAID decision tree based on training-database; CHAID-T, CART decision tree based on training-database; CHAID-V, the } \\
\text { validation of CHAID; CART-V, the validation of CART }\end{array}$
\end{tabular}


Table 2

Predictions of CHAID and CART for the outcome to discriminate NE and SKC in training database

\begin{tabular}{|c|c|c|c|c|}
\hline Node & Discriminating rules & Outcome & Probability(\%) & Population \\
\hline \multicolumn{5}{|c|}{ CHAID decision tree in the training-database } \\
\hline 4 & If (BAD-D >1.94) & SKC & 100 & 25 \\
\hline 3 & If $(1.53<$ BAD-D $\leq 1.94)$ & SKC & 84.6 & 11 \\
\hline 5 & If (BAD-D $\leq 0.92)$ and (DA-ratio $\leq 4.5)$ & NE & 91.1 & 41 \\
\hline 7 & If $(0.92<$ BAD-D $\leq 1.53)$ and $(S P A 1 \leq 92.2)$ & SKC & 88.9 & 8 \\
\hline 9 & If $(0.92<$ BAD-D $\leq 1.53),($ SPA1 $>92.2)$ and $(B e c c \leq 0.60)$ & NE & 95 & 19 \\
\hline \multicolumn{5}{|c|}{ CART decision tree in the training-database } \\
\hline 2 & If $(B A D-D>1.635)$ & SKC & 100 & 34 \\
\hline 3 & If (BAD-D $\leq 1.635)$ and $(\mathrm{SPA} 1 \leq 89.25)$ & SKC & 91.7 & 11 \\
\hline 5 & If (BAD-D $\leq 1.635)$, (SPA1 >89.25), and (Becc $\leq 0.605)$ & NE & 91.9 & 57 \\
\hline 7 & If (BAD-D $\leq 1.635),($ SPA1 >89.25), (Becc >0.605), and (PPImax $\leq 1.25)$ & NE & 90 & 9 \\
\hline 8 & If (BAD-D $\leq 1.635),($ SPA1 >89.25), (Becc >0.605) and $($ PPImax >1.25) & SKC & 70 & 7 \\
\hline
\end{tabular}

Table-3. Descriptive statistics of the key studied variables in the training-database

\begin{tabular}{|c|c|c|c|c|c|c|c|c|c|}
\hline Variable & NE & SKC & $P$ value & AUC & $\begin{array}{l}95 \% \\
\mathrm{Cl}\end{array}$ & Cutoff & Accuracy & Sensitivity & Specificity \\
\hline BAD-D & $0.79 \pm 0.4$ & $2.04 \pm 1.00$ & $<0.001^{*}$ & 0.86 & $\begin{array}{l}0.79- \\
0.92\end{array}$ & 1.33 & $82.0 \%$ & $69.0 \%$ & $92.9 \%$ \\
\hline SPA1 & $111.9(103.5,121.6)$ & 89.3(81.0,100.8) & $<0.001^{\#}$ & 0.86 & $\begin{array}{l}0.78- \\
0.91\end{array}$ & 101.2 & $81.3 \%$ & $79.3 \%$ & $82.9 \%$ \\
\hline DA-ratio & $4.3(4.0,4.4)$ & $4.9(4.5,5.2)$ & $<0.001^{\#}$ & 0.84 & $\begin{array}{l}0.77- \\
0.90\end{array}$ & 4.5 & $78.9 \%$ & $70.7 \%$ & $85.7 \%$ \\
\hline PPImax & $1.24(1.15,1.32)$ & $1.48(1.34,1.63)$ & $<0.001^{\#}$ & 0.86 & $\begin{array}{l}0.78- \\
0.91\end{array}$ & 1.34 & $82.0 \%$ & $74.1 \%$ & $88.6 \%$ \\
\hline Becc & $0.49(0.43,0.56)$ & $0.60(0.48,0.65)$ & $0.001^{\#}$ & 0.68 & $\begin{array}{l}0.59- \\
0.76\end{array}$ & 0.54 & $67.2 \%$ & $63.8 \%$ & $70.0 \%$ \\
\hline CBI & $0.01(0,0.04)$ & $0.18(0.02,0.81)$ & $<0.001^{\#}$ & 0.80 & $\begin{array}{l}0.72- \\
0.87\end{array}$ & 0.05 & $77.3 \%$ & $65.5 \%$ & $87.1 \%$ \\
\hline TBI & $0.07(0.01,0.18)$ & $0.35(0.20,1.00)$ & $<0.001^{\#}$ & 0.84 & $\begin{array}{l}0.76- \\
0.90\end{array}$ & 0.17 & $78.1 \%$ & $81.0 \%$ & $75.7 \%$ \\
\hline \multicolumn{10}{|c|}{$\begin{array}{l}\text { BAD-D, Belin/Ambrósio Enhanced Ectasia Display Index; SPA1, stiffness parameter at first applanation; DA-ratio, deformation } \\
\text { amplitude ratio; PPImax, maximum of pachymetric progression index; Becc, eccentricity of back surface; CBI, corvis } \\
\text { biomechanical index; TBI, combined tomography and biomechanical index; }\end{array}$} \\
\hline \multicolumn{10}{|c|}{ * Independent t-test. \# Mann-Whitney U test } \\
\hline
\end{tabular}


Table-4. Delong's Test Results of Major Predictors' AUCs for NE vs SKC

\begin{tabular}{|lllll|}
\hline & AUC difference & SE & $95 \%$ Cl & P value \\
\hline BAD-D vs CBI & 0.06 & 0.04 & $-0.02-0.15$ & 0.158 \\
\hline BAD-D vs TBI & 0.02 & 0.03 & $-0.04-0.09$ & 0.420 \\
\hline TBI vs CBI & 0.04 & 0.05 & $-0.06-0.13$ & 0.422 \\
\hline CHAID-T vs BAD-D & 0.07 & 0.03 & $0.01-0.13$ & 0.014 \\
\hline CHAID-T vs CBI & 0.14 & 0.04 & $0.05-0.22$ & 0.001 \\
\hline CHAID-T vs TBI & 0.10 & 0.03 & $0.03-0.16$ & 0.004 \\
\hline CART-T vs BAD-D & 0.08 & 0.03 & $0.02-0.14$ & 0.015 \\
\hline CART-T vs CBI & 0.14 & 0.05 & $0.05-0.23$ & 0.002 \\
\hline CART-T vs TBI & 0.10 & 0.03 & $0.04-0.17$ & $\mathbf{0 . 0 0 2}$ \\
\hline CHAID-T vs CART-T & 0.01 & 0.02 & $-0.03-0.05$ & 0.781 \\
\hline $\begin{array}{l}\text { BAD-D, Belin/Ambrósio Enhanced Ectasia Display Index; CBI, corvis biomechanical index; TBI, combined tomography and } \\
\text { biomechanical index; CHAID-T, CHAID decision tree based on training database; CHAID-T, CART decision tree based on training } \\
\text { database }\end{array}$ & & & \\
\hline
\end{tabular}

Descriptive statistics of the key studied variables are displayed in Table 3. Compared with those in NE eyes, the BAD-D, DA ratio, PPImax, Becc, CBI, and TBI were significantly higher, and the SPA1 was significantly lower in SKC eyes. The ROC results showed that the BAD-D, SPA1 and PPImax had the highest AUC values of 0.86 , followed by the DA-ratio and TBI (AUC values: 0.84 and 0.84 , respectively). The AUCs of the CHAID-T and CART-T were 0.94 and 0.94 , respectively. The CHAID-V and CART-V demonstrated similar AUC values to those in the training database (CHAID-V, 0.92; CART-V, 0.95). DeLong's test showed that both the CHAID-T and CART-T demonstrated significantly higher AUC values than each AUC of the individual BAD-D, CBI, and TBI (Table 4). The ROC curves of the CHAID-T, CART-T, BAD-D, CBI, and TBI are presented in Figure 3A, whereas the ROC curves of the CHAID-V and CART-V are presented in Figure 3B.

\section{Discussion}

We developed two DT models separately based on the CHAID and CART methods in a training database. The results showed that the CART-T model had a higher discriminating accuracy of $92.2 \%$ ( $89.7 \%$ sensitivity and $94.3 \%$ specificity), whereas the CHAID-T model demonstrated $88.3 \%$ accuracy with $82.8 \%$ sensitivity and $92.9 \%$ specificity. The nonparametric nature of a DT allows for the lack of requirement for a specified distribution of outcome data or a large sample size; however, this nature renders them more sensitive to overfitting than parametric models. ${ }^{16}$ To minimize the overfitting risk, we performed cross-validation in both the CHAIDT and CART-T procedures. Based on the same database, internal validation itself may still overestimate model performance; therefore, an independent database is recommended to evaluate the model for generalizability. In the external validation procedure, we found that the CART-V had a similar predictive accuracy for SKC compared to the CART-T (Table 1). Compared with that of CHAID-T, the sensitivity of CHAID-V increased, whereas the specificity and overall predictive accuracy decreased. In terms of sensitivity and specificity, the performance of the CART in the training and validation databases presented high consistency. Considering the severe outcomes of missed diagnosis of SKC, the CART-T with higher predictive sensitivity and generalizability may be an optimal tool for ophthalmologists to detect SKC.

Based on different tree-growing methods, both CHAID-T and CART-T selected BAD-D as the most discriminant variable to distinguish between the SKC and NE. Studies of SKC screening have confirmed the superior discriminatory power of the BAD-D over topographic variables and central thickness evaluation. ${ }^{17,18}$ The cutoff values of the BAD-D used to discriminate SKC from NE were 1.6 in Shetty's study ${ }^{18}$ and 1.09 in Steinberg's study ${ }^{8}$, whereas the cutoff value of the individual BAD-D was 1.33 in our study. When selecting an optimal cutoff value, special attention should be given to the generalizability of it found within the studied population. In the present study, both the CHAID-T and CART-T selected similar cutoff values (1.53 in CHAID-T and 1.635 in CART-T) of BAD-D to 
generate the first split, which were nearly equal to the reference value of 1.6 between normal and suspected patients provided by the Pentacam system. Selecting a higher cutoff value of the BAD-D resulted in high screening specificity for SKC while decreasing the false-positive rate in the first split of the DTs. Accumulating these findings, BAD-D should be most considered in the clinical diagnostic rule of SKC.

Both DTs selected biomechanical variables as the second most discriminant variable for differentiating SKC from NE (CHAID with DA-ratio and SPA1; CART with SPA1). Compared with the CHAID-T with multiple branches, the CART-T with a binary split nature incorporated a lower cutoff value of the SPA1 with 89.25 (SPA1 cutoff of 92.2 in CHAID-T). Generally, the CART tree-growing method attempts to maximize within-node homogeneity. Zhou reported that the SPA1 index, which reflects corneal stiffness, decreased and had a significant correlation with the thinnest corneal thickness in moderate-to-severe KC but not in NE. ${ }^{19}$ The DA ratio reflects the corneal deformation level under specific air impulses; a weaker cornea is more prone to deformation. Previous studies have demonstrated that the individual DA ratio and SPA1 can effectively discriminate KC from NE but performed worse in discriminating SKC from NE. ${ }^{20,21}$ In our study, both the individual DA ratio and SPA1 demonstrated the same AUC of 0.84 when comparing SKC and NE. However, the results in this study highlighted the importance of biomechanical variables in SKC diagnosis.

According to our findings, PPImax and Becc were not selected in the upper part of either the CHAID-T or CART-T. The PPImax represents the corneal thickness distribution from the thinnest point to the periphery, and the PPImax increases if the central cornea becomes thinner. It has been validated with higher accuracy for discriminating $\mathrm{KC}$ from NE than that of the single pachymetric index. ${ }^{22}$ We found that the PPImax had an AUC value of 0.86 for discriminating SKC from NE. Eccentricity is an important index for describing changes in corneal shape, and the normal value ranges from $0.4-0.6 .{ }^{23} \mathrm{~A}$ previous study reported that eccentricity increases in keratoconus, even in its primary stages, with normal visual acuity of 1.0 and normal slit-lamp findings. ${ }^{24}$ Although the AUC of individual Becc was low for differentiating SKC from NE in our study, collaboration with other variables in the DTs strengthened its function in SKC screening.

When comparing the performance of the DTs with other models, a major problem that arises is the inconformity of studied populations among different studies. Smadja reported that a DT based on Scheimpflug imaging parameters had a higher accuracy of $96.4 \%$ than that of our discriminating rules for detecting SKC. ${ }^{5}$ In fact, stricter criteria (normal topography and BED) were used for SKC patient selection in our study. Atalay et al. developed a logistic regression model that combined metrics from tomography and ocular response analyzer imaging and reported that the model could detect SKC with $87.1 \%$ sensitivity and $91.4 \%$ specificity 25 . In their study, the inclusion criterion for SKC considered BED; however, they included individuals who fell into the suspected category $(B E D \leq 16 \mu \mathrm{m})$. Regardless of the difference in inclusion criteria, the predictive accuracy of DT models in our study was higher than that of the logistic-regression model in Atalay's study.

Interestingly, using the same SKC criteria of (KISA\% $<60 \%$ ) and (I-S value $<1.45 \mathrm{D}$ ), the AUCs of individual BAD-D, CBI, and TBI were variant between Ambrósio's study (AUCs for BAD-D,0.84; CBI, 0.82; TBI, 0.99) ${ }^{6}$ and Steinberg's study (AUCs for BAD-D,0.75; CBI, 0.79; $\mathrm{TBI}, 0.83)^{8}$ when comparing SKC and NE. According to the correlation between the KISA\% index and BAD-D reported by Steinberg ${ }^{26}$, we robustly converted the BAD-D value of each individual in our study to $\mathrm{KISA} \%$ and found that all included patients met the criteria of KISA\% $<60 \%$. Both Steinberg's study ${ }^{8}$ and our study found that there were no significant differences between any two AUCs among those of the individual BAD-D, CBI, and TBI for differentiating SKC and NE. Furthermore, DeLong's test showed that both the CHAID-T and CART-T had significantly higher AUCs than those of the individual BAD-D, CBI, and TBI. These findings confirmed that the performance of DT models in detecting SKC was superior to that of the summary combined metrics. However, to identify the superiority of the DT, further studies based on different methodologies using our training and validation databases are required.

To investigate earlier changes in SKC, previous studies considered BAD-D analysis in the selection of SKC patients, which resulted in lower cutoffs with consuming sensitivity and specificity. 8,12 In our study, we did not consider BAD-D analysis when selecting SKC patients. First, the BAD-D has been reported to have good performance in SKC screening, and we desired that the studied model include the efficient parameter BAD-D. Second, the strictest criteria with a normal BAD-D may result in a lower cutoff in the DT discriminating rules and diminish the generalizability of the screening model in the whole population.

In conclusion, the DT models combined with metrics from tomography and biomechanical imaging showed excellent performance for discriminating SKC with normal topography and BED from NE, which highlighted the importance of overall analysis from 
broader features of the cornea. Moreover, the DT models generated visualized and easy-to-understand discriminating rules to help ophthalmologists screen for SKC. The BAD-D variable was the most critical determinant in classifying and identifying SKC, followed by SPA1.

\section{Declarations}

Acknowledgments: This study was supported by the Health and Family Planning Committee Science Foundation of Wuhan, WX18Q14 and the Science Research Foundation of Aier Eye Hospital Group, AF1904D3, AU1904D1, and AR1904D3.

Author Contributions: Q.Y. Z and S.W. R contributed to the design of the study and confirmation of patient recruitment. P. S contributed to the manuscript preparation and data analysis. $Y$. $L$ and $P$. $L$ contributed to the examination and data collection. All authors reviewed the manuscript.

Informed Consent Statement: Written informed consent was obtained from all individual participants.

included in the study.

Data Availability Statement: The data presented in this study are available on request from the corresponding author.

Conflicts of Interest: The authors declare no competing financial interests.

Additional Information: This original research has not been previously published or presented in any meeting previously.

\section{References}

1. Sharif, R., Bak-Nielsen, S., Hjortdal, J. \& Karamichos, D. Pathogenesis of Keratoconus: The intriguing therapeutic potential of Prolactin-inducible protein. Prog Retin Eye Res, 67, 150-167 (2018).

2. Shajari, M. et al. Early Tomographic Changes in the Eyes of Patients With Keratoconus. J Refract Surg, 34 (4), 254-259 (2018).

3. Bae, G. H. et al. Corneal topographic and tomographic analysis of fellow eyes in unilateral keratoconus patients using Pentacam. Am J Ophthalmol, 157 (1), 103-109101 (2014).

4. Hwang, E. S., Perez-Straziota, C. E., Kim, S. W., Santhiago, M. R. \& Randleman, J. B. Distinguishing Highly Asymmetric Keratoconus Eyes Using Combined Scheimpflug and Spectral-Domain OCT Analysis., 125 (12), 1862-1871 (2018).

5. Smadja, D. et al. Detection of subclinical keratoconus using an automated decision tree classification. Am J Ophthalmol, 156 (2), 237-246231 (2013).

6. Ambrosio, R. Jr. et al. Integration of Scheimpflug-Based Corneal Tomography and Biomechanical Assessments for Enhancing Ectasia Detection. J Refract Surg, 33 (7), 434-443 (2017).

7. Rabinowitz, Y. S. \& Rasheed, K. KISA\% index: a quantitative videokeratography algorithm embodying minimal topographic criteria for diagnosing keratoconus. J Cataract Refract Surg, 25 (10), 1327-1335 (1999).

8. Steinberg, J. et al. Tomographic and Biomechanical Scheimpflug Imaging for Keratoconus Characterization: A Validation of Current Indices. J Refract Surg, 34 (12), 840-847 (2018).

9. Gomes, J. A. et al. Global consensus on keratoconus and ectatic diseases., 34 (4), 359-369 (2015).

10. Muftuoglu, O., Ayar, O., Ozulken, K., Ozyol, E. \& Akinci, A. Posterior corneal elevation and back difference corneal elevation in diagnosing forme fruste keratoconus in the fellow eyes of unilateral keratoconus patients. J Cataract Refract Surg, 39 (9), 1348-1357 (2013).

11. Temstet, C. et al. Corneal epithelial thickness mapping using Fourier-domain optical coherence tomography for detection of form fruste keratoconus. J Cataract Refract Surg, 41 (4), 812-820 (2015).

12. Koc, M. et al. Biomechanical Analysis of Subclinical Keratoconus With Normal Topographic, Topometric, and Tomographic Findings. J Refract Surg, 35 (4), 247-252 (2019).

13. Koc, M. et al. An Early Finding of Keratoconus: Increase in Corneal Densitometry., 37 (5), 580-586 (2018).

14. Vinciguerra, R. et al. Detection of Keratoconus With a New Biomechanical Index. J Refract Surg, 32 (12), 803-810 (2016).

Page $8 / 12$ 
15. Chan, T. C. Y., Wang, Y. M., Yu, M. \& Jhanji, V. Comparison of Corneal Tomography and a New Combined Tomographic Biomechanical Index in Subclinical Keratoconus. J Refract Surg, 34 (9), 616-621 (2018).

16. Altman, D. G., Vergouwe, Y., Royston, P. \& Moons, K. G. Prognosis and prognostic research: validating a prognostic model. BMJ, 338, b605 (2009).

17. Song, P. et al. Assessment of Corneal Pachymetry Distribution and Morphologic Changes in Subclinical Keratoconus with Normal Biomechanics. Biomed Res Int, 2019, 1748579 (2019).

18. Shetty, R. et al. Keratoconus Screening Indices and Their Diagnostic Ability to Distinguish Normal From Ectatic Corneas. Am J Ophthalmol, 181, 140-148 (2017).

19. Zhao, Y. et al. Relationship Among Corneal Stiffness, Thickness, and Biomechanical Parameters Measured by Corvis ST, Pentacam and ORA in Keratoconus. Front Physiol, 10, 740 (2019).

20. Chan, T. C., Wang, Y. M., Yu, M. \& Jhanji, V. Comparison of corneal dynamic parameters and tomographic measurements using Scheimpflug imaging in keratoconus. Br J Ophthalmol, 102 (1), 42-47 (2018).

21. Kataria, P. et al. Jr. Accuracy of Scheimpflug-derived corneal biomechanical and tomographic indices for detecting subclinical and mild keratectasia in a South Asian population. J Cataract Refract Surg, 45 (3), 328-336 (2019).

22. Ambrosio, R. Jr. et al. Novel pachymetric parameters based on corneal tomography for diagnosing keratoconus. J Refract Surg, 27 (10), 753-758 (2011).

23. Vinciguerra, P. \& Camesasca, F. I. Treatment of hyperopia: a new ablation profile to reduce corneal eccentricity. J Refract Surg, 18 (3 Suppl), S315-317 (2002).

24. Dao, C. L., Kok, J. H., Brinkman, C. J. \& van Mil, C. J. Corneal eccentricity as a tool for the diagnosis of keratoconus., 13 (4), 339-344 (1994).

25. Atalay, E., Ozalp, O., Erol, M. A., Bilgin, M. \& Yildirim, N. A Combined Biomechanical and Tomographic Model for Identifying Cases of Subclinical Keratoconus., 39 (4), 461-467 (2020).

26. Steinberg, J. et al. Correlation of the KISA\% index and Scheimpflug tomography in 'normal', 'subclinical', 'keratoconus-suspect' and 'clinically manifest' keratoconus eyes. Acta Ophthalmol, 93 (3), e199-207 (2015).

\section{Figures}




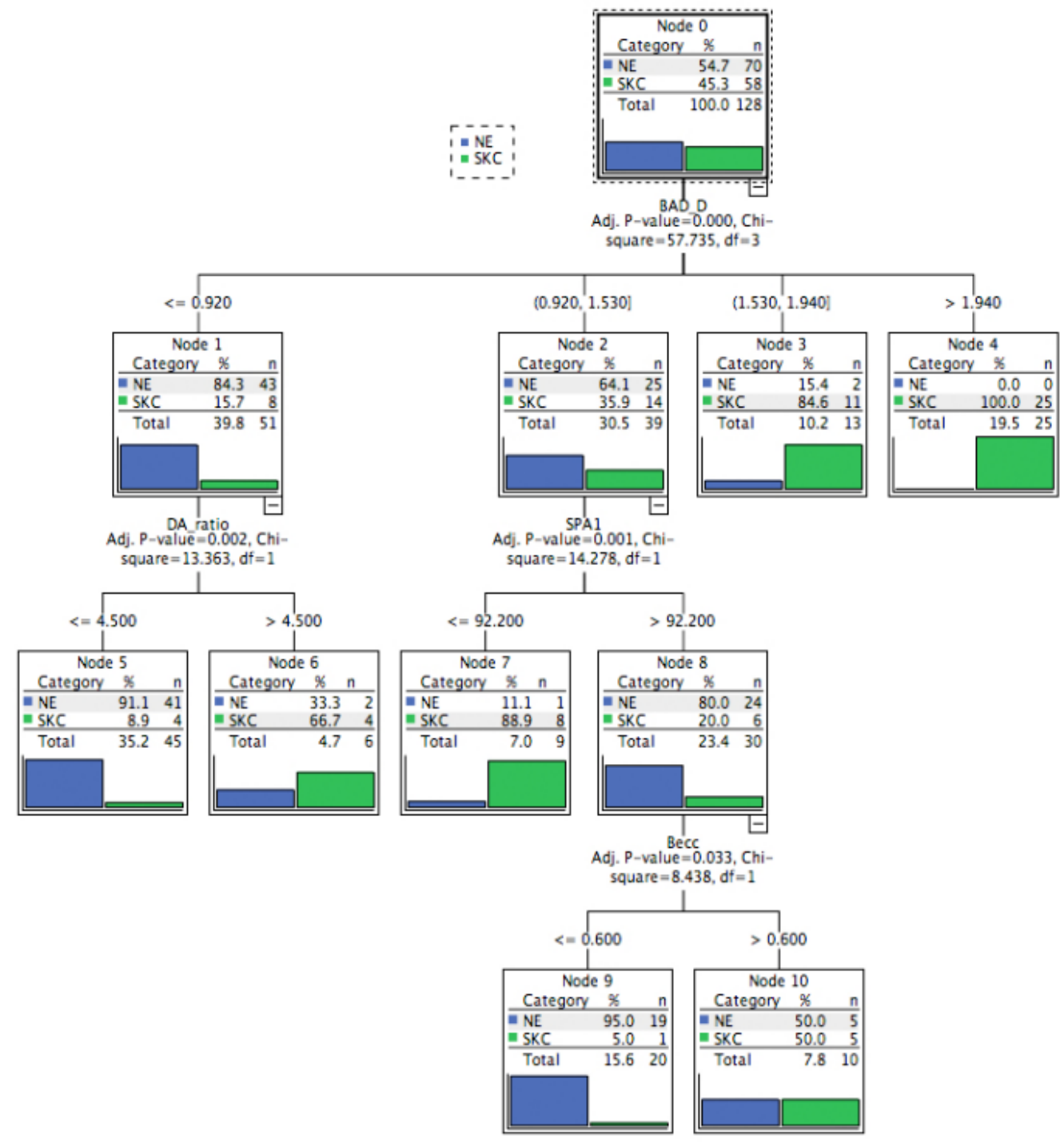

\section{Figure 1}

The CHAID decision tree to discriminate between NE and SKC in the training database (CHAID-T). This discriminating rule achieved $82.8 \%$ sensitivity and $92.9 \%$ specificity. When generating a split, the cutoff values were as follows: BAD-D: $0.92,1.53$, and 1.94; DAratio, 4.5; SPA1, 92.2; and Becc, 0.6. Details of the splitting between categories of eyes are specified in the boxes at each node. The category with the gray background was the targeted category. CHAID, Chi-square automatic interaction detection; NE, normal eye; SKC, subclinical keratoconus; CHAID-T, CHAID tree in the training database; BAD-D, Belin/Ambrósio deviation index; DA ratio, deformation amplitude ratio; SPA1, stiffness parameter at first applanation; Becc, back eccentricity 


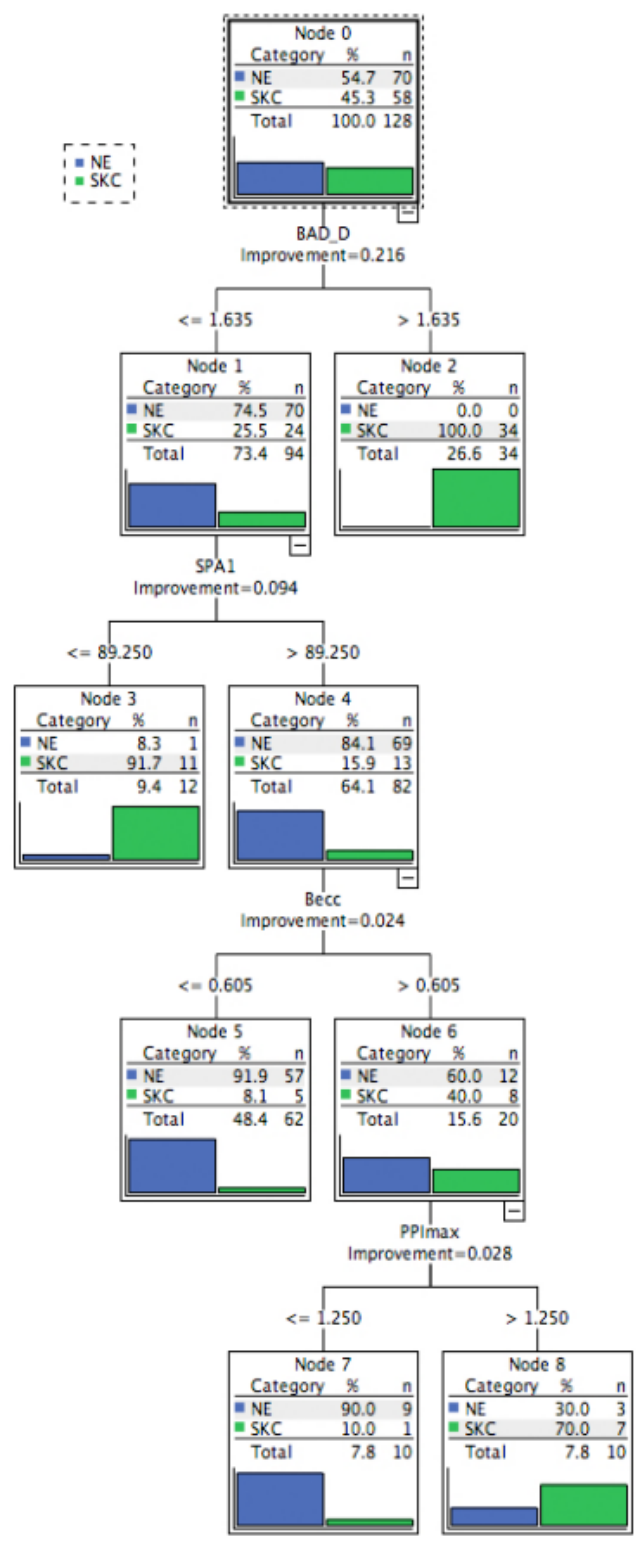

\section{Figure 2}

The CART decision tree for discriminating between NE and SKC in the training database (CART-T). This discriminating rule achieved $89.7 \%$ sensitivity and $94.3 \%$ specificity. When generating a split, the cutoff values were as follows: BAD-D, $1.635 ;$ SPA1, 89.25; Becc, 0.605; and PPImax, 1.25. Details of the splitting between categories of eyes are specified in the boxes at each node. The category with the gray background was the targeted category. CART, classification and regression tree; NE, normal eye; SKC, subclinical keratoconus; CART-T, CART tree in the training database; BAD-D, Belin/Ambrósio deviation index; SPA1, stiffness parameter at first applanation; Becc, back eccentricity; PPImax, maximum pachymetric progression index 

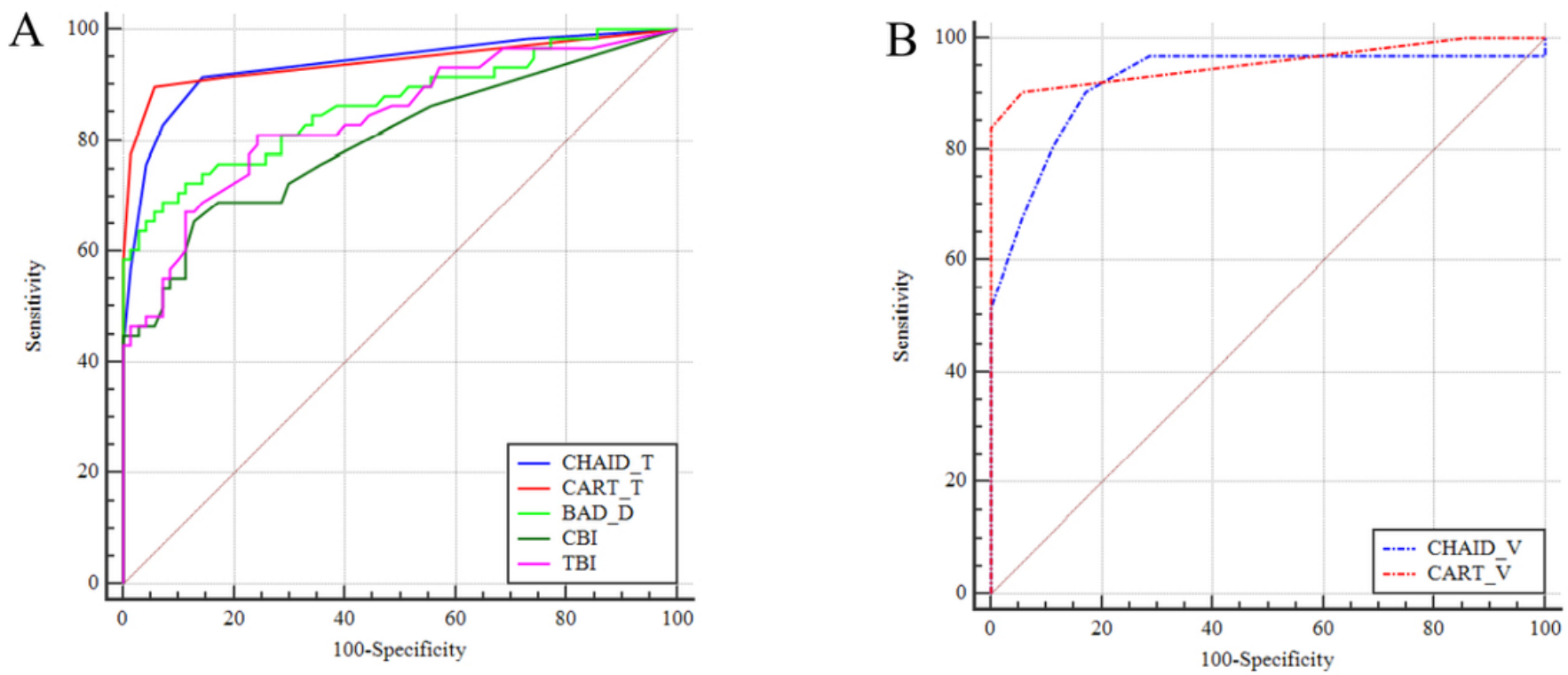

\section{Figure 3}

The ROC curves of the CHAID-T, CART-T, BAD-D, CBI, and TBI are presented in (A), whereas the ROC curves of decision trees in the validation database are presented in (B). ROC, receiver operating characteristic; CHAID-T, CHAID tree in the validation database; CART-T, CART tree in the validation database; BAD-D, Belin/Ambrósio deviation index; CBI, Corvis biomechanical index; TBI, tomographic and biomechanical index

\section{Supplementary Files}

This is a list of supplementary files associated with this preprint. Click to download.

- SupplementaryFigure1Legend.docx

- SupplementaryFigure1.pdf 